\title{
INFLUENCIA DEL TRATAMIENTO UV-C SOBRE EL TIEMPO DE VIDA ÚTIL Y PROPIEDADES ANTIOXIDANTES DE PRODUCTOS DE IV GAMA (MÍNIMAMENTE PROCESADOS) DE CARAMBOLA (Averrhoa cambola L.)
}

\author{
Autores: \\ Bioq. María José Andrade Cuvi' \\ Ing. Carlota Moreno Guerrero' \\ Dra. Analía Concellón ${ }^{2}$
}





\section{Resumen}

Se estudió la influencia del tratamiento UV-C sobre el tiempo de vida útil y propiedades antioxidantes enzimáticas y no enzimáticas de productos IV Gama de carambola (Averrhoa carambola L.). Frutos mínimamente procesados se dividieron en dos grupos: frutos tratados $\left(13 \mathrm{~kJ} / \mathrm{m}^{2}\right)$ y no tratados(controles) y se almacenaron en refrigeración durante 21 días. A los 7, 14 y 21 días se determinó la pérdida de peso, acidez, $\mathrm{pH}$, sólidos solubles, pérdida de electrolitos, azúcares totales, color superficial y el avance de pardeamiento, decaimiento y firmeza al tacto. Se realizaron análisis microbiológicos de mohos y aerobios totales, se cuantificó el contenido de fenoles totales, flavonoides, vitamina C, capacidad antioxidante total y la actividad enzimática de superóxido dismutasa-SOD, catalasa-CAT y peroxidasa-POX. No se observaron diferencias significativas en los parámetros físico-químicos. El tratamiento con radiación UV-C retrasó la aparición de síntomas de daño, redujo la pérdida de electrolitos y el crecimiento de microorganismos, produjo acumulación de fenoles totales y flavonoides y mayor capacidad antioxidante. Se produjeron cambios en las actividades enzimáticas de SOD, CAT y POX. Probablemente, los cambios producidos por el tratamiento UV-C podrían relacionarse con el incremento del tiempo de vida útil del producto, sin embargo es necesario realizar un estudio más detallado.

Palabras clave: carambola mínimamente procesada, radiación UV-C, postcosecha, antioxidantes.

\section{Abstract}

The influence of UV-C treatment on shelf life and antioxidant enzymatic and nonenzymatic IV product range carambola (Averrhoa carambola L.). Minimally processed fruits were divided into two groups: treated fruits $\left(13 \mathrm{~kJ} / \mathrm{m}^{2}\right)$ and untreated (control) and stored under refrigeration for 21 days. At 7, 14 and 21 days was determined by weight loss, acidity, pH, soluble solids, loss of electrolytes, total sugars, surface color and the progress of browning, decay and firmness to the touch. Microbiological analysis were made from molds and aerobic total quantified content of total phenols, flavonoids, vitamin C, total antioxidant capacity and enzyme activity of superoxide dismutase-SOD, catalase-CAT and peroxidase-POX. There were no significant differences in physical and chemical parameters. Treatment with UV-C delayed the onset of symptoms of damage, reduced loss of electrolytes and the growth of microorganisms, produced accumulation of total phenolics and flavonoids and highest antioxidant capacity. There were changes in enzyme activities of SOD, CAT and POX. Probably, changes caused by UV-C treatment could be associated with increased lifetime of the product, however it is necessary to conduct a more detailed study.

Key words: star fruit minimally process, irradiation UV-C, postharvest, antioxidants 


\section{Introducción}

La carambola (Averrhoa carambola L.) es una fruta exótica, subtropical, altamente perecedera, de origen asiático, perteneciente a la familia de las oxalidáceas, muy cotizada en mercados internacionales, introducida en el Ecuador hace 30 años, conocida popularmente como "fruta estrella" o "star fruit". Tiene forma ovalada, alargada, con cinco aristas o alas y, al corte, de estrella de cinco puntas (figura 1). Es de pequeño tamaño, con una longitud que oscila entre 7 y $12 \mathrm{~cm}$, tiene una piel fina, lustrosa y comestible, de color entre verde o dorado y amarillo-anaranjado cuando está madura. La pulpa es crujiente, de suave textura y amarilla vidriosa; la pulpa tiene pocas o ninguna semilla, es abundante, crujiente, jugosa y con un fino sabor agridulce. También es utilizada para decoración y cocina gourmet. No se recomienda su almacenamiento a temperaturas menores a $5^{\circ} \mathrm{C}$.

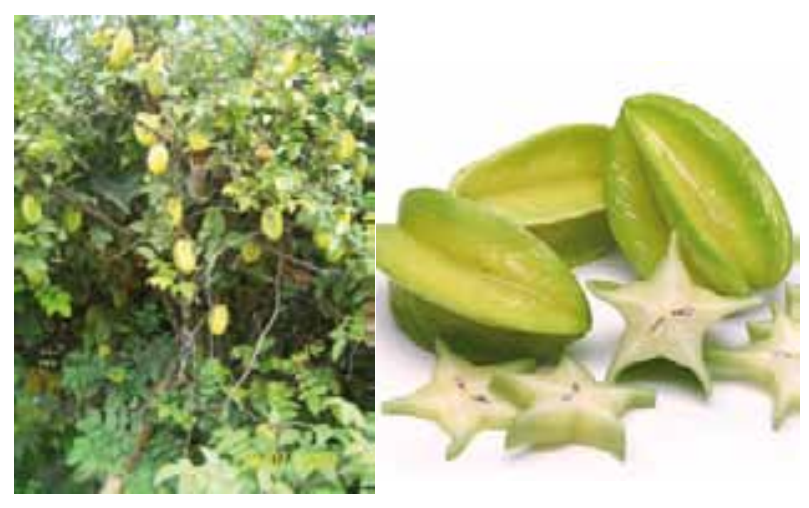

Figura 1. Carambola (Averrhoa carambola L.)

En Ecuador, la carambola se cultiva principalmente en la zona subtropical. El rendimiento de producción promedio es de $28000-$ $32000 \mathrm{~kg} / \mathrm{Ha}$ (SIGAGRO, 2002). Se consume como fruta entera o se usa para la elaboración de vinos artesanales y decoración de platos en la cocina gourmet. Por sus características, la fruta entera es susceptible a sufrir daños por magulladuras y ataque de mohos y una vez cortada presenta rápido pardeamiento, reduciendo su vida útil.
Por otro lado, las frutas y hortalizas mínimamente procesadas en fresco (IV Gama), constituyen un sector de rápido crecimiento en la industria de los alimentos. Las operaciones propias del procesamiento mínimo incluyen: lavado del producto entero, deshojado, pelado, deshuesado, cortado, lavado y desinfectado. Una vez que los productos se procesan, se empaquetan en bolsas selladas o en bandejas cubiertas con plásticos, con o sin atmósfera modificada para, posteriormente, ser almacenados y transportados bajo refrigeración (Lobo y González, sin fecha). Las modificaciones de calidad más importantes que sufren se deben a la presencia de superficies cortadas y tejidos vegetales dañados, a que dicho proceso no puede asegurar la esterilización o la estabilidad microbiológica del producto y a que, su metabolismo sigue estando activo. Así, las reacciones de degradación que se producen afectan a cualidades organolépticas tales como el color, firmeza, aroma, sabor y valor nutricional (Lobo y González, sin fecha).

Con el fin de alargar el tiempo de vida útil de productos mínimamente procesados, en los últimos años se han estudiado tecnologías postcosecha como atmósferas modificadas, aplicación de ozono, recubrimiento comestibles, radiación gamma, radiación UV-C, etc.

Se ha demostrado la efectividad del tratamiento con radiación UV-C en productos IV GAMA como mango (González-Aguilar y col., 2007), melón (Lamikanra y col., 2005), tuna (Piga y col; 1998), brócoli (Costa y col., 2006), pimiento (Vicente y col., 2005), entre otros, siendo una alternativa tecnológica en la postcosecha de frutos exóticos enteros y mínimamente procesados.

La radiación UV-C (254 nm) posee una importante acción bactericida y por otro lado, se propone su uso para producir un efecto beneficioso en los tejidos en respuesta a dosis bajas o subletales según el concepto de "hormesis" (Luckey, 1980); de esta forma, el uso de la radiación UV-C se perfila como una de las tecnologías con mayor aplicación en el futuro. Cisneros-Zevallos (2003) sugiere la aplicación 
postcosecha de un tipo de estrés abiótico controlado (por ejemplo la exposición a luz UV-C) para inducir la producción e incremento de la síntesis de compuestos fotoquímicos con actividad nutracéutica o la reducción de compuestos indeseables. Así el control del estrés inducido por la luz UV-C puede usarse como una herramienta para reforzar las propiedades benéficas de productos frescos enteros o cortados.

Se ha reportado que la exposición a dosis bajas de UV-C retrasó la maduración y senescencia en manzana (Liu y col., 1991), mango (González-Aguilar y col., 2001), durazno (González-Aguilar y col., 2004) y naranja (D'hallewin y col., 1999); sin embargo pocos estudios se han realizado para elucidar el modo de acción de la radiación UV-C sobre estos sistemas. Barka y col., (2000) estudiaron los efectos de la luz UV-C en la inducción de algunas enzimas en tomate y encontraron una significativa peroxidación lipídica, pérdida de electrolitos a través de membrana, además reportaron la activación de enzimas involucradas en mecanismos de defensa como la fenilalanina amonioliasa y lipooxigenasa (Barka, 2001) y la inducción de enzimas relacionadas al estrés oxidativo como guayacol peroxidasa y ascorbato peroxidasa.

Todos los seres vivos, como parte del metabolismo generan especies reactivas de oxígeno (EROs) tales como hidroperóxido, radical hidroxilo, oxígeno singulete y triplete, radical superóxido. Su producción y eliminación están reguladas por un sistema antioxidante de tipo enzimático (por ejemplo superóxido dismutasa-SOD-, catalasa -CAT- y peroxidasa -POX-) y no enzimático (compuestos químicos, por ej. Vitamina C). Cuando existe un desequilibrio entre la producción y neutralización o eliminación de las EROs, éstas se acumulan en la célula pudiendo producir daño a moléculas de $A D N$, lípidos y proteínas, provocando un estado de estrés oxidativo (Mittler, 2002). Se le atribuye a este estado la producción de enfermedades como cáncer, diabetes, enfermedades cardiovasculares, entre otras. De ahí la importancia del consumo diario de alimentos ricos en compuestos antioxidantes. Una de las mayores fuentes de antioxidantes son los vegetales (Cisneros-Zevallos, 2003).
Con estos antecedentes, se hace necesaria más evidencia experimental para elucidar el modo de acción de esta tecnología aplicada a productos hortifructícolas. El objetivo del presente estudio fue estudiar la influencia del tratamiento UV-C sobre el tiempo de vida útil y propiedades antioxidantes enzimáticas y no enzimáticas de productos IV Gama (mínimamente procesados) de carambola (Averrhoa carambola L.).

Como hipótesis se planteó que el tratamiento UV-C incrementa el tiempo de vida útil y mantiene y/o mejora las propiedades antioxidantes de carambola (Averrhoa carambola L.) mínimamente procesada.

\section{Materiales y Métodos}

\section{Material Vegetal y selección de la dosis efectiva de radiación} UV-C

Frutos de carambola (Averroha carambola L.) fueron cosechados en la provincia de Manabí (Ecuador) y trasladados inmediatamente al laboratorio, una vez lavados y seleccionados, se cortaron manualmente rebanadas de $5 \mathrm{~mm}$ de ancho.

Para determinar las condiciones experimentales adecuadas se ensayaron diferentes dosis de luz UV-C. Los frutos se dividieron en dos grupos: control (no irradiados) y tratados (irradiados). Estos últimos se colocaron bajo cuatro lámparas UV-C (TUV G30T8, 30W, Philips) a una distancia de $30 \mathrm{~cm}$ y fueron irradiados con dosis de 6,10 y $13 \mathrm{~kJ} / \mathrm{m}^{2}$. La intensidad de la radiación fue medida con un radiómetro digital UV (UVP Modelo Deluxe.). Cada rebanada fue rotada manualmente para asegurar una exposición uniforme a la luz UV en toda su superficie. Finalizado el tratamiento los frutos se colocaron en bandejas plásticas cubiertos con un film de PVC y se almacenaron a $5^{\circ} \mathrm{C}$ durante 21 días. Los controles (sin ningún tratamiento) se empacaron con la misma película y almacenaron directamente a $5^{\circ} \mathrm{C}$. Se retiraron de la cámara de almacenamiento a los 0, 7, 14 y 21 días para evaluar el efecto de 
cada tratamiento según el avance del daño mediante el valor de índice de daño, pérdida de peso y análisis microbiológico. La experiencia completa se realizó por duplicado.

Una vez elegida la dosis de $13 \mathrm{~kJ} / \mathrm{m}^{2}$ se efectuó el tratamiento sobre frutos (como se indicó anteriormente) y se los almacenó junto a los frutos control a $5^{\circ} \mathrm{C}$ por 0, 7, 14 y 21 días. A cada día de muestreo los frutos fueron retirados de la cámara de almacenamiento y luego de estimar el índice de daño, medir el color superficial y pérdida de peso, realizar los análisis físico-químicos y microbiológicos, se retiraron las semillas y la piel, se cortaron y congelaron en $\mathrm{N}_{2}$ líquido para finalmente conservarlos a $-20^{\circ} \mathrm{C}$ hasta su análisis bioquímico.

2. Efecto de la radiación sobre la pérdida de peso, índice de daño y parámetros físico-químicos y microbiológicos.

2.1 Pérdida de peso.- Se pesó cada bandeja al inicio y al término de cada período de almacenamiento. Se determinó la pérdida de peso como porcentaje del peso inicial.

2.2 Índice de daño.- Cada día de muestreo los frutos se evaluaron visualmente utilizando una escala subjetiva, considerando los siguientes parámetros: decaimiento, pardeamiento y firmeza al tacto. Usando índice de daño (ID) con una escala subjetiva de 1 a 4 , donde: $4=$ daño severo, 3 =daño moderado, 2 =daño ligero y $1=\sin$ daño.

2.3 Sólidos solubles totales.- Se determinaron en el jugo de carambola, mediante un refractómetro de mano marca Atago.

2.4 pH.- Se midió en el jugo de carambola de cada muestra, con un potenciómetro digital marca Metter Toledo 320.

2.5 Acidez titulable como \%Ácido cítrico.- Se titularon $5 \mathrm{~mL}$ de extracto de jugo obtenido con $\mathrm{NaOH}(0,1 \mathrm{~N})$, utilizando fenolftaleína $(0,1 \%)$ como indicador.
2.6 Pérdida de electrolitos.- Se midió según el método de Concellón y col. (2005) con modificaciones, 10 discos de pulpa de la

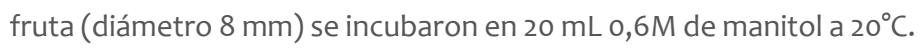
La conductividad de la solución fue medida al inicio de la incubación y dos horas después usando un conductímetro marca ThermoOrion. Para evaluar la cantidad residual de electrolitos remanentes en el tejido, se trituraron los discos de tejido, se filtró y se midió la conductividad del filtrado. Los resultados se expresaron como porcentaje de electrolitos remanentes en el tejido luego de 2 horas. Las medidas se realizaron por triplicado.

2.7 Azúcares totales.- El contenido de azúcares totales expresados como porcentaje se determinó por espectrofotometría según el método MO-L-SAIA-2T, realizado en el Instituto Nacional Autónomo de Investigaciones Agropecuarias (INIAP-Santa Catalina Quito-Ecuador) a donde se trasladaron las muestras congeladas a $-20^{\circ} \mathrm{C}$ para su análisis.

2.8 Color superficial.- Fue evaluado con un colorímetro (Marca TMP ${ }^{\circledR}$-México) por medición de los parámetros L, H, y S. Se realizaron tres medidas en cada superficie de la rebanada.

2.9 Análisis microbiológico.- El recuento de levaduras y mohos se realizó mediante el método $\mathrm{AOAC}^{\circledR}$ Official Method ${ }^{\mathrm{SM}} 997.02$ para alimentos, método film seco rehidratable (Petrifilm).

\section{Efecto de la radiación UV-C sobre el contenido de compuestos antioxidantes.}

3.1 Contenido de Fenoles totales (FT) y Flavonoides (Flav). Se trituró el tejido y homogeneizó en etanol. El contenido de fenoles totales fue medido usando el reactivo de Folin \& Ciocalteau (Singelton y Rossi, 1965), mientras que, para flavonoides se usó el método de Shin y col. (2007). Se empleó catequina como estándar. 
3.2 Cuantificación de vitamina C.- A cada día de muestreo se extrajo el jugo de fruta y se midió el contenido de vitamina $C$ expresado como $\mathrm{mg} / \mathrm{L}$, se determinó por reflectometría según el método MO-L-SAIA-10, realizado en el Instituto Nacional Autónomo de Investigaciones Agropecuarias (INIAP-Santa Catalina QuitoEcuador) a donde se trasladaron las muestras de jugo congelado a $-20^{\circ} \mathrm{C}$ para su análisis.

3.3 Capacidad antioxidante total.- Se analizó utilizando el radical estable 2,2-difenil-1-picrilhidrazil (DPPH•) en etanol (BrandWilliams y col., 1995) y se realizó la lectura espectrofotométrica a $517 \mathrm{~nm}$. Los resultados se expresan como $\mu$ equivalente Trolox/g tejido.

4 Efecto de la radiación UV-C sobre la actividad de enzimas antioxidantes.

4.1 Preparación de los extractos.- Se tomaron $2 \mathrm{~g}$ de tejido, se congeló en $\mathrm{N} 2$ líq., se pulverizó y adicionó $10 \mathrm{ml}$ de buffer fosfato

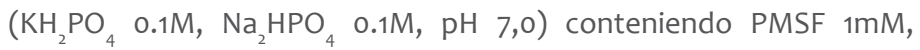
EDTA $0.1 \mathrm{mM}$ y PVPP $30 \mathrm{~g} / \mathrm{L}$. Se agitó, se centrifugó, se separó el sobrenadante, se filtró, se distribuyó en alícuotas que se almacenaron a $-20^{\circ} \mathrm{C}$ hasta ser usadas.

4.2 Medida de la actividad enzimática.

4.2.1 Actividad Superóxido dismutasa (SOD). Fue determinada midiendo el grado de inhibición de la reducción del NBT en presencia de riboflavina a 560nm según el método de Giannopolitis y Ries (1977). La mezcla de reacción contenía 100 mM de buffer $\mathrm{Na}_{2} \mathrm{HPO}_{4}$ y $\mathrm{KH}_{2} \mathrm{PO}_{4} 100 \mathrm{mM}$, pH 7.8, metionina $13 \mathrm{mM}$, nitroblue tetrazolium (NBT) $75 \mu \mathrm{M}$, riboflavina $6 \mu \mathrm{M}$, EDTA 0,1 mM y $300 \mu \mathrm{L}$ de extracto enzimático. La mezcla de reacción fue expuesta a $30 \mathrm{~cm}$ bajo lámparas fluorescentes (120W) por 15 min. La reacción empieza/ termina con el encendido/apagado de las lámparas, respectivamente. Una mezcla de reacción irradiada, sin extracto enzimático se utilizó como control. Una mezcla completa de reacción no irradiada fue usada como blanco. Una unidad de actividad de SOD se define como la cantidad de enzima que, bajo las condiciones de ensayo, causa un 50\% de inhibición de la reacción (o absorbancia) observada en ausencia de enzima.

4.2.2 Actividad Catalasa. La actividad CAT se determinó según el método de Sinha (1972) con modificaciones, cuantificando la descomposición de $\mathrm{H}_{2} \mathrm{O}_{2}$ no consumido, a través de la reacción con $\mathrm{K}_{2} \mathrm{Cr}_{2} \mathrm{O}_{7}$ y ácido acético, formando un compuesto de color verde, estable. La mezcla de reacción contenía buffer fosfato de potasio 100mM, pH 7.0, $\mathrm{H}_{2} \mathrm{O}_{2} 800 \mathrm{mM}$, y $800 \mu \mathrm{L}$ de extracto enzimático. Alícuotas de $200 \mu \mathrm{L}$ se tomaron a los o y 5 min y la reacción se detuvo por adición de éstas a $1 \mathrm{~mL}$ de $\mathrm{K}_{2} \mathrm{Cr}_{2} \mathrm{O}_{7}(5 \%)$ y ácido acético glacial. Se midió la absorbancia de las muestras a 570nm a temperatura ambiente. Se efectuó una curva de calibración de $\mathrm{H}_{2} \mathrm{O}_{2}$ entre 60 y 200 mM. Una unidad de actividad de CAT fue definida como el consumo de $\mathrm{H}_{2} \mathrm{O}_{2} /($ min. g tejido fresco). Las determinaciones se realizaron por triplicado.

4.2.3 Actividad Peroxidasa. La actividad POX fue estimada según el método de Flurkey y Je, (1978), con ligeras modificaciones. La mezcla de reacción contenía buffer fosfato de potasio $100 \mathrm{mM}, \mathrm{pH}$ 7.0, $300 \mu \mathrm{L}$ de extracto enzimático, $500 \mu \mathrm{L}$ guayacol $0,25 \%(\mathrm{v} / \mathrm{v})$ y $160 \mu \mathrm{L}$ de $\mathrm{H}_{2} \mathrm{O}_{2} 100 \mathrm{mM}$ con un volumen final de $2000 \mu \mathrm{L}$. Se cuantificó el incremento de la absorbancia a 470nm, durante 2 min a temperatura ambiente. Una unidad de actividad de POX fue definida como el cambio en la absorbancia/(min . g tejido fresco)

\subsection{Análisis estadístico.}

Se empleó un diseño factorial. Los resultados fueron procesados mediante un ANOVA y las medidas comparadas por el test LSD con un $\alpha=0,05$. 


\section{Resultados y Discusión}

1. Selección de la dosis efectiva de radiación UV-C

Según se observa en la Tabla 1, la pérdida de peso de los frutos control fue de $3,8 \%$ con respecto al día inicial del almacenamiento. En los frutos tratados con las dosis de 6 y $10 \mathrm{~kJ} / \mathrm{m}^{2}$, fue 8,7 y $9,8 \%$ respectivamente, mientras que los frutos tratados con $13 \mathrm{~kJ} / \mathrm{m}^{2}$ sufrieron la menor pérdida de peso (4,7\%).

También se encontró que los frutos control presentaron síntomas de daño a los 7 días de almacenamiento, diferenciándose de los frutos tratados que mantenían una mejor calidad. Aún a los 14 días de almacenamiento los frutos tratados presentaron mejores características con respecto a los frutos control. A los 21 días de almacenamiento, los frutos control habían perdido totalmente su calidad alcanzando un valor de ID de 3,7; en cambio, los frutos tratados con 6,10 y $13 \mathrm{~kJ} / \mathrm{m}^{2}$ alcanzaron valores de ID de 2,6; 2,5 y 2,3 respectivamente.

Tabla 1. Análisis microbiológicos, pérdida de peso (\%), e índice de daño en frutos control y tratados $\left(6,10\right.$ y $\left.13 \mathrm{~kJ} / \mathrm{m}^{2}\right)$ durante 21 días de almacenamiento a $5^{\circ} \mathrm{C}$.

\begin{tabular}{|c|c|c|c|c|c|c|}
\hline \multirow{2}{*}{\multicolumn{2}{|c|}{ Parámetro }} & \multirow{3}{*}{$\begin{array}{c}\text { Tiempo } \\
\text { (Días) } \\
0\end{array}$} & \multirow{3}{*}{\begin{tabular}{|c|} 
Control \\
3,11
\end{tabular}} & \multicolumn{3}{|c|}{ Dosis UV-C } \\
\hline & & & & \multirow{2}{*}{$\begin{array}{c}\mathbf{6} \mathbf{~ k J} / \mathbf{~ m}^{\mathbf{2}} \\
3,41\end{array}$} & \multirow{2}{*}{$\begin{array}{c}10 \mathbf{~ k J} / \mathbf{~ m}^{2} \\
1,48\end{array}$} & \multirow{2}{*}{$\begin{array}{c}13 \mathbf{~ k J} / \mathbf{~ m}^{2} \\
1,00\end{array}$} \\
\hline \multirow{4}{*}{$\begin{array}{l}\text { Análisis } \\
\text { microbiológicos }\end{array}$} & \multirow{2}{*}{$\begin{array}{l}\text { Mohos } \\
(\log \text { UPM/g) }\end{array}$} & & & & & \\
\hline & & 21 & 4,71 & 5,95 & 4,79 & 4,54 \\
\hline & \multirow{2}{*}{$(\log U F C / g)$} & 0 & 3,43 & 2,61 & 4,51 & 4,58 \\
\hline & & 21 & 6,62 & 4,53 & 4,72 & 4,72 \\
\hline \multicolumn{2}{|l|}{ Pérdida peso (\%) } & 21 & 3,8 & 8,7 & 9,8 & 4,7 \\
\hline \multicolumn{2}{|l|}{ Índice de Daño } & 21 & 3,7 & 2,3 & 2,5 & 2,6 \\
\hline
\end{tabular}

En cuanto al análisis microbiológico, las dosis de 10 y $13 \mathrm{~kJ} / \mathrm{m}^{2}$ retrasaron el crecimiento de mohos y aerobios totales con respecto a la dosis de $6 \mathrm{~kJ} / \mathrm{m}^{2}$ y las muestras control (datos no mostrados). Por lo tanto, la dosis de $13 \mathrm{~kJ} / \mathrm{m}^{2}$ fue seleccionada para los ensayos posteriores.

\begin{abstract}
2. Efecto de la radiación UV-C $\left(13 \mathrm{~kJ} / \mathrm{m}^{2}\right)$ sobre la pérdida de peso, índice de daño y parámetros físico-químicos y microbiológicos.
\end{abstract}

2.1. Pérdida de peso.

Los frutos tratados presentaron un promedio de 2,8\% de pérdida de peso luego de 21 días de almacenamiento y no se observó diferencia significativa entre las muestras control y tratadas, resultados similares fueron reportados en brócoli mínimamente procesado tratado con radiación UV-C (Lemoine y col., 2007).

\subsection{Indice de daño.}

En la figura 2, se expone la variación en los ID de los frutos en función del tiempo de almacenamiento.

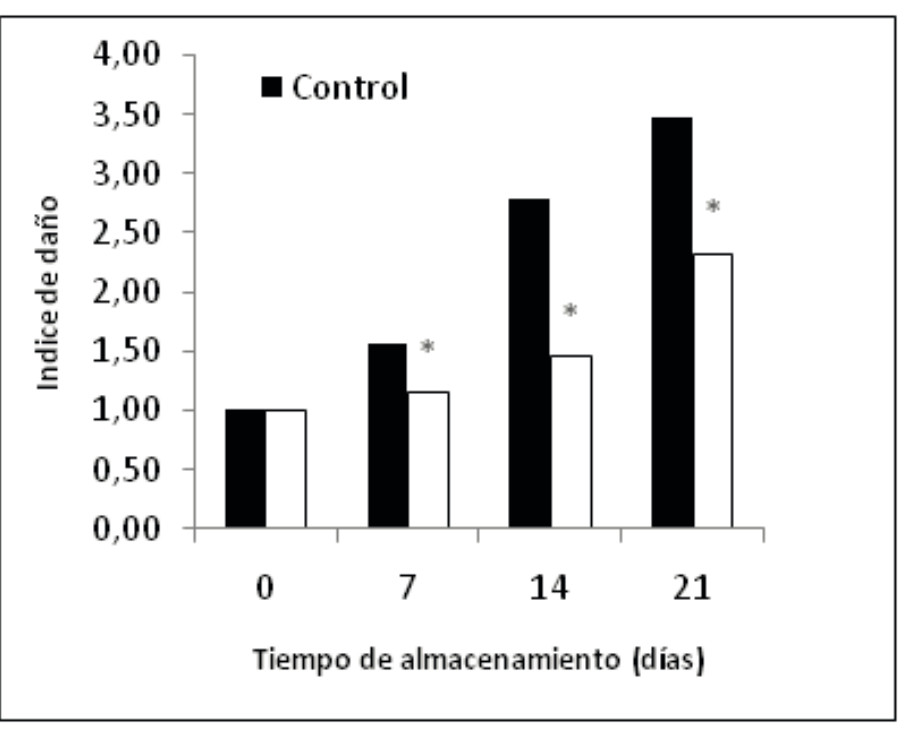

Figura 2. Variación del índice de daño de carambola fresca cortada en función del tiempo de almacenamiento a $5^{\circ} \mathrm{C}$. El asterisco indica que el valor es significativamente diferente del correspondiente control con una $\mathrm{p}<0.05$

Se evidenció daño en los frutos control a partir del día 7, alcanzando un daño moderado a severo $(I D=3,5)$ en el día 21 perdiendo totalmente su calidad organoléptica y comercial, mientras 
que los frutos tratados alcanzaron al final del almacenamiento un daño de leve a moderado (ID=2,7) manteniendo una mejor calidad organoléptica y comercial (figura 3).
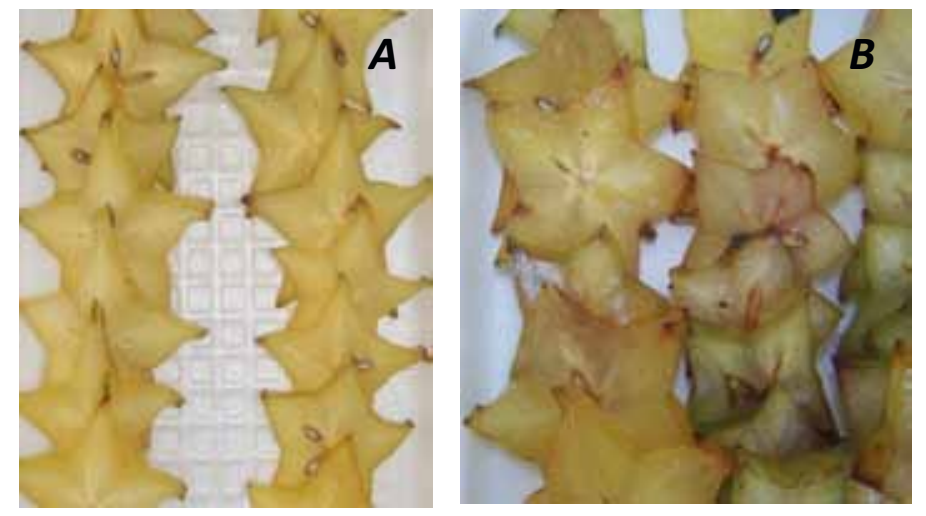

Figura 3. Desarrollo del índice de daño en frutos de carambola fresca cortada tratada (A) con $13 \mathrm{~kJ} / \mathrm{m}^{2}$ y controles (B) luego de 21 días de almacenamiento a $5^{\circ} \mathrm{C}$.

2.3. $\mathrm{pH}$, sólidos solubles totales y acidez titulable total. Durante el período de almacenamiento no se observó variación en las características químicas tanto de los frutos tratados como de los frutos control, tampoco se evidenció diferencia significativa entre las muestras (datos no mostrados), estos resultados indicarían que la dosis de radiación UV-C $\left(13 \mathrm{~kJ} / \mathrm{m}^{2}\right)$ usada en este experimento, que se puede considerar baja, probablemente no afectaría la integridad del tejido.

\subsection{Pérdida de electrolitos}

La cuantificación de la pérdida de electrolitos puede tomarse como una medida indirecta que indica pérdida de la integridad del tejido, ya que como se ha reportado en frutos como la berenjena (Concellón y col., 2005) la pérdida de electrolitos se incrementa en forma paralela con la aparición de síntomas de daño.

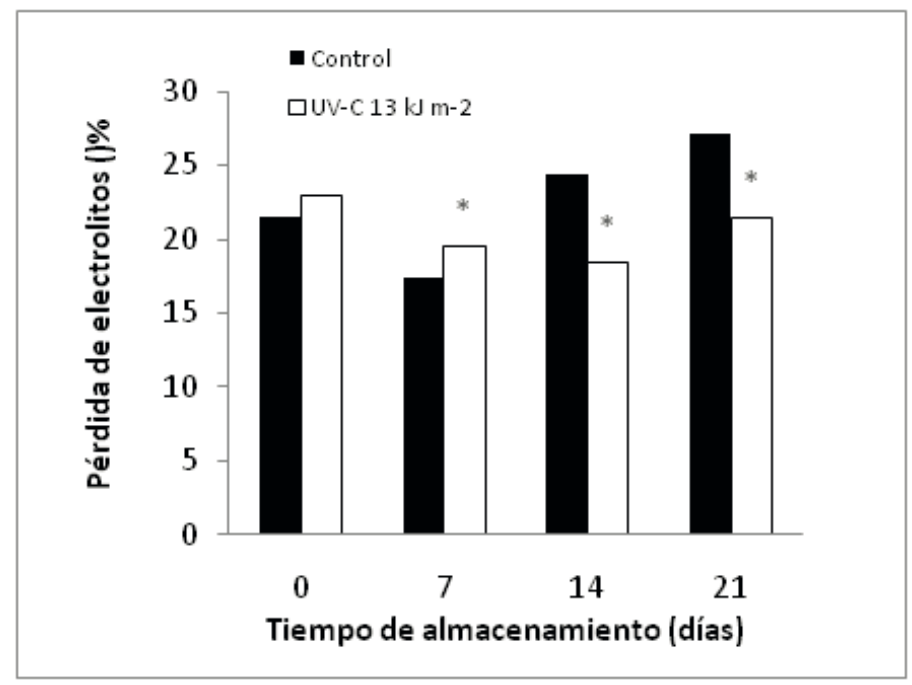

Figura 4. Pérdida de electrolitos en carambola fresca cortada en función del tiempo de almacenamiento a $5^{\circ} \mathrm{C}$. El asterisco indica que el valor es significativamente diferente del correspondiente control con una $\mathrm{p}<0.05$.

En la carambola mínimamente procesada se observó un incremento en la pérdida de electrolitos en los frutos control (figura 4) a lo largo del almacenamiento, mientras que en los frutos tratados presentaron menor pérdida de electrolitos. Estos resultados sugieren que la radiación UV-C permitiría mantener la integridad del tejido por más tiempo, es decir menos daño del tejido. Resultados similares han sido descritos por Vicente y col., (2005) en pimiento tratado con radiación UV-C.

\subsection{Azúcares totales}

Inmediatamente después de la aplicación de la radiación UV-C el contenido de azúcares totales se redujo en los frutos tratados con respecto a los controles. Un comportamiento similar al observado en el presente estudio fue reportado por Pan y col. (2004) al aplicar radiación UV-C en frutillas.Durante el almacenamiento (figura 5), los frutos controles mantuvieron estable la concentración de azúcares totales, mientras que los frutos tratados presentaron mayor concentración de azúcares totales que los controles a partir del día 7 . 
Al no observarse cambios en el contenido de azúcares totales y en la acidez total titulable de la fruta, factores que influyen en el sabor y en la aceptación del consumidor, el tratamiento con luz UV-C permitiría mantener la calidad de la fruta por más tiempo.

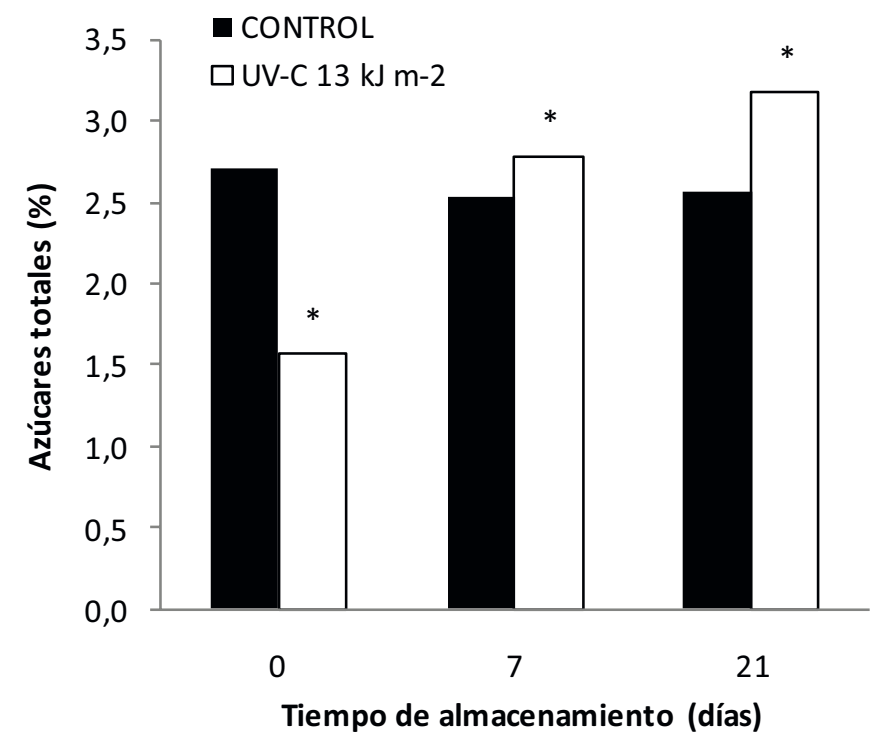

Figura 5. Variación del contenido de azúcares totales (\%) en carambola fresca cortada en función del

tiempo de almacenamiento a $5^{\circ} \mathrm{C}$. El asterisco indica que el valor es significativamente diferente del

correspondiente control con una $\mathrm{p}<0.05$.

En pimiento entero (Vicente y col., 2005) y en zuquini mínimamente procesado (Erkan y col., 2001) el tratamiento con radiación UV-C no produjo diferencia en el contenido de azúcares totales entre los frutos tratados y controles, a diferencia de los resultados encontrados en el presente estudio. La variación del contenido de azúcares totales en el tejido puede deberse a múltiples razones por lo que se hace necesaria la investigación para elucidar el comportamiento del tejido frente la radiación UV-C.

\subsection{Color superficial}

Para la medición del efecto de la radiación UV-C sobre el color se utilizó la escala de color HSL, un modelo basado en la percepción humana en donde, $\mathrm{H}$ indica el tono del color, $\mathrm{S}$ indica el grado de separación del gris neutral y L el porcentaje de blanco a negro. Como se puede observar en la Tabla 2, la tonalidad del color (H) se modificó mayormente en los frutos controles que en los tratados, es decir se observó un mayor cambio de amarillo verdoso a amarillo, que podría indicar el desarrollo del color durante la maduración del fruto, sugiriendo que la radiación UV-C retrasaría este desarrollo de color. En cuanto al parámetro $\mathrm{S}$, los frutos control presentaron mayores valores que los tratados, lo que podría indicar que éstos últimos tienen menor oscurecimiento en el tejido. Mientras que al evaluar la luminosidad (L) se observó que los frutos tratados mantuvieron el color del tejido más claro que los controles ya que a menores valores de L, existe mayor oscurecimiento. Resultados similares fueron reportados por Pan y col., (2001).

\begin{tabular}{|lccccc|}
\hline $\begin{array}{l}\text { Parámetros } \\
\text { de color }\end{array}$ & Muestra & \multicolumn{5}{c}{ Tiempo de almacenamiento (días) } \\
\hline $\mathbf{H}$ & & $\mathbf{0}$ & $\mathbf{7}$ & $\mathbf{1 4}$ & $\mathbf{2 1}$ \\
\hline LSD = 1,37 & $\mathrm{C}$ & 37,00 & 30,22 & 27,72 & 14,50 \\
\cline { 2 - 6 } & $\mathrm{T}$ & $33,34^{*}$ & $26,94^{*}$ & 29,09 & $26,50^{*}$ \\
\hline $\mathbf{S}$ & & & & & \\
\hline LSD $=\mathbf{2 , 1 7}$ & $\mathrm{C}$ & 13,46 & 25,91 & 30,37 & 45,55 \\
\hline L & $\mathrm{T}$ & $20,39^{*}$ & 29,68 & $26,48^{*}$ & $32,63^{*}$ \\
\hline LSD $=\mathbf{1 , 4 2}$ & $\mathrm{C}$ & 29,43 & 25,93 & 17,07 & 17,23 \\
\cline { 2 - 6 } & $\mathrm{T}$ & 32,42 & $21,93^{*}$ & $22,35^{*}$ & $21,20^{*}$ \\
\hline
\end{tabular}

Tabla 2. Variación de los parámetros de color H (tono de color), S

(saturación), L (luminosidad) en

frutos control y tratados $(13 \mathrm{KJ} / \mathrm{m} 2)$ durante el tiempo de almacenamiento. El asterisco indica que

el valor es significativamente diferente del correspondiente control con una $\mathrm{p}<0.05$ Para cada caso se presenta el valor de la diferencia mínima significativa (LSD) con un $\mathrm{p}<0,05$

\subsection{Análisis microbiológicos.}

Para el control de microorganismos en alimentos usualmente se utiliza el escaldado o pasteurización, en los últimos años la radiación UV-C, una tecnología no térmica, se ha aplicado en el procesamiento de alimentos para inactivar varios tipos de microorganismos (Guerrero-Beltrán y col., 2004). En esta investigación se observó 
que a partir del día 14 los frutos control dejaron de ser consumibles dado que mostraban desarrollo de microorganismos (figura 6). Los frutos tratados recién mostraron desarrollo fúngico a los 21 días de almacenamiento a $5^{\circ} \mathrm{C}$. De la misma forma se ha comprobado la eficacia de la radiación UV-C sobre el control de Botrytis cinerea en fresa (Marquenie y col., 2002), en melón entero y procesado (Lamikanra y col., 2005), control de hongos en pimiento (Vicente y col. 2005), entre otros.

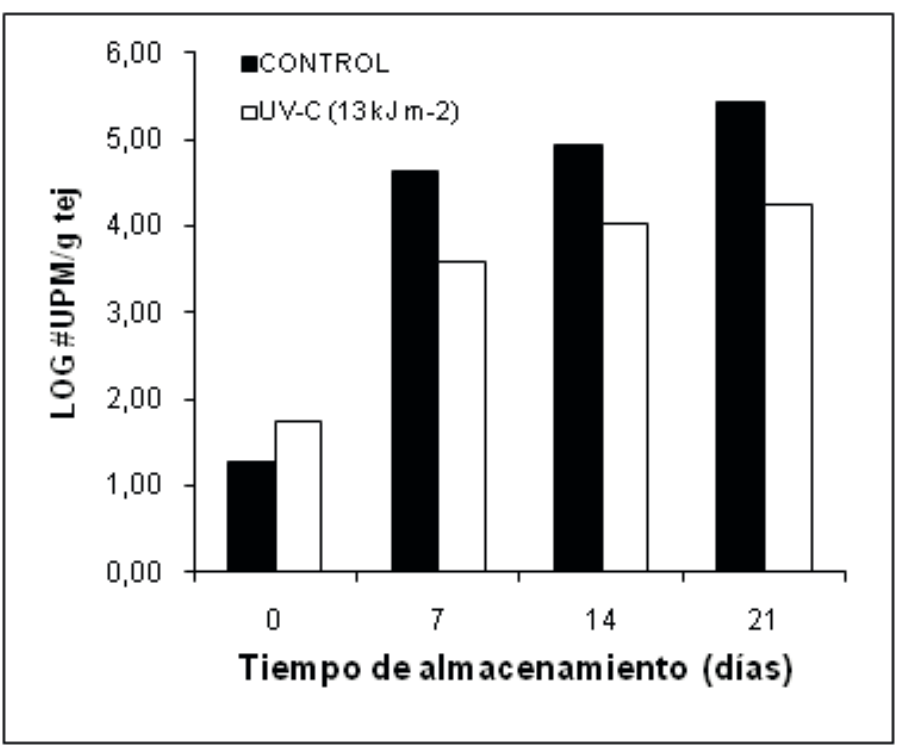

Figura 6. Decaimiento en frutos control y tratado lo largo del almacenamiento refrigerado $\left(5^{\circ} \mathrm{C}\right)$

3. Efecto de la radiación UV-C $\left(13 \mathrm{~kJ} / \mathrm{m}^{2}\right)$ sobre Compuestos antioxidantes.

3.1. Contenido de fenoles totales y flavonoides. Los compuestos fenólicos son sustancias involucradas en el mantenimiento de la capacidad antioxidante de un tejido. En los frutos tratados, a partir del día inicial del experimento se presentó un incremento gradual hasta el final del almacenamiento. A diferencia de los frutos controles, en los que el contenido de FT se mantuvo prácticamente constante durante este período y en menor concentración que los frutos tratados (figura 7). De la misma forma, se observó un incremento del contenido de fenoles totales en pimiento (Vicente y col., 2005), y brócoli (Costa y col., 2006) tratados con una dosis de UV-C de 7 y $10 \mathrm{~kJ} /$ $\mathrm{m}^{2}$ respectivamente; al igual que en mangos enteros y mango fresco cortado expuestos a luz UV-C (González-Aguilar y col., 2007).

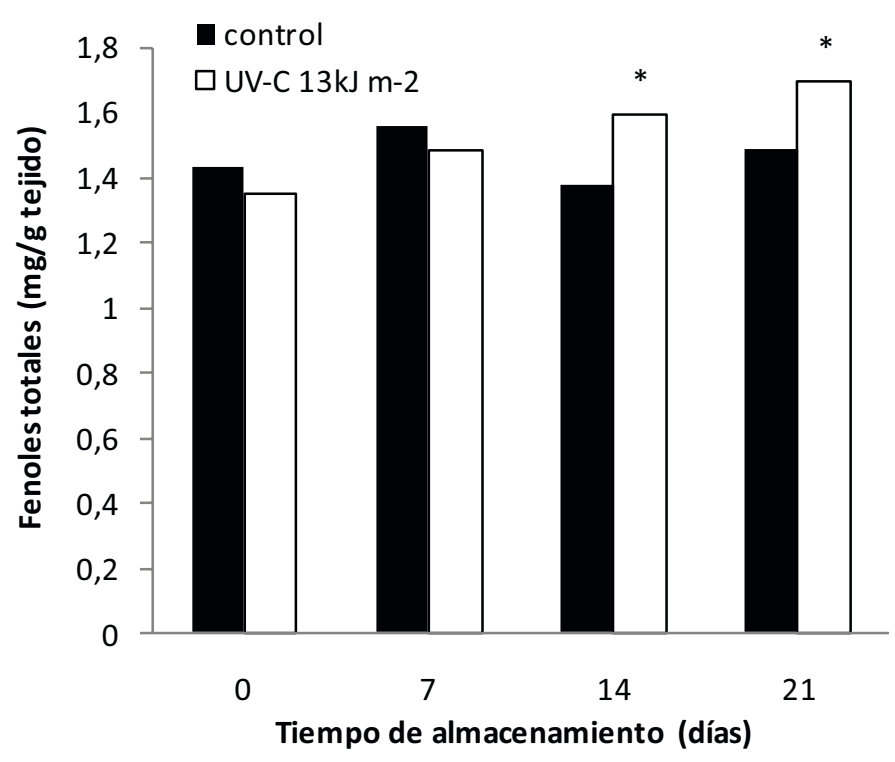

Figura 7. Contenido de fenoles totales en carambola mínimamente procesada control y tratada $\left(13 \mathrm{~kJ} / \mathrm{m}^{2}\right)$ durante 21 días de almacenamiento a $5^{\circ} \mathrm{C}$. El asterisco indica que el valor es significativamente diferente del correspondiente control con una $\mathrm{p}$ $<0.05$.

Inmediatamente después del tratamiento se observó mayor concentración de Flav en los frutos tratados que en los controles (figura 8). En el día 7, los frutos tratados presentaron disminución en el contenido de Flav, a partir del cual se mantuvo constante hasta el final del almacenamiento, los frutos controles presentaron un comportamiento similar que los tratados, sin embargo la pérdida de Flav fue menor. 


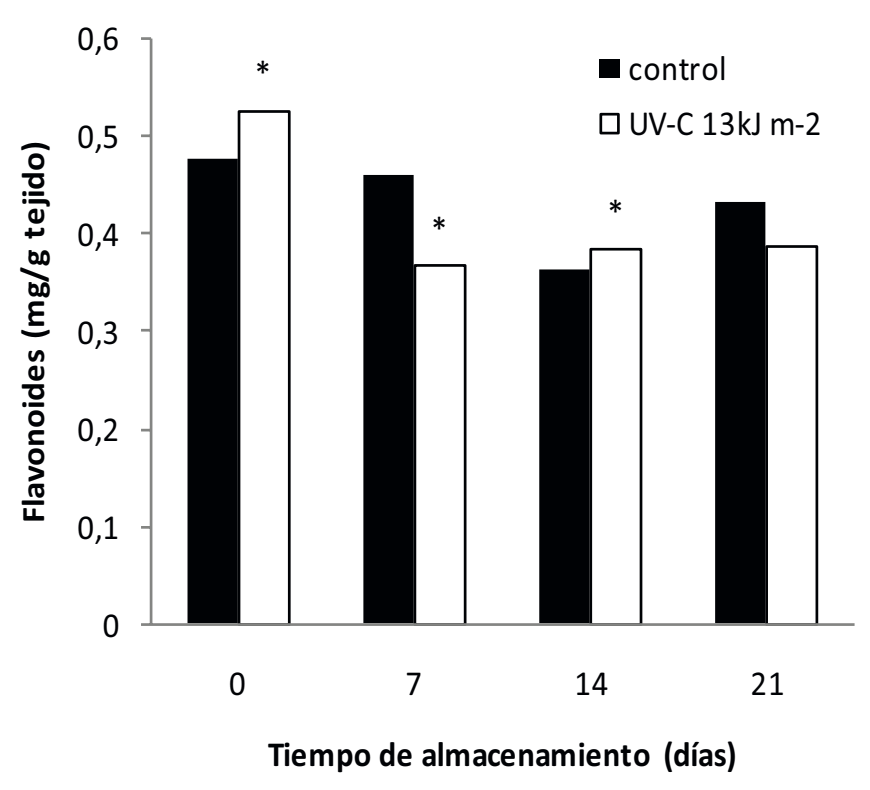

Figura 8. Contenido de flavonoides en carambola mínimamente procesada control y tratada $\left(13 \mathrm{~kJ} / \mathrm{m}^{2}\right)$ durante 21 días de almacenamiento a $5^{\circ} \mathrm{C}$. El asterisco indica que el valor es significativamente diferente del correspondiente control con una $\mathrm{p}$ $<0.05$.

Si bien las muestras control presentaron mayor contenido de Flav, este valor no influyó en el contenido de FT debido a que corresponde aproximadamente al 20\% de los FT, resultados similares se encontraron en estudios de pimiento tratado con $10 \mathrm{~kJ} / \mathrm{m}^{2}$ (Andrade-Cuvi, 2008). El incremento de compuestos con actividad antioxidante se asocia con la tolerancia o retraso en la aparición de daño en los tejidos (Wang, 1995).

\subsection{Vitamina $C$}

Si bien la aplicación de la radiación UV-C induce la acumulación de sustancias con capacidad antioxidante como los polifenoles, es poco conocido el efecto de esta tecnología sobre la retención de ácido ascórbico (González-Aguilar y col., 2007; Erkan y col., 2001). Al aplicar una dosis de $13 \mathrm{~kJ} / \mathrm{m}^{2}$ en carambola mínimamente procesada se produjo un efecto negativo, es decir la pérdida de vitamina $C$ durante el almacenamiento. Resultados similares fueron reportados por
González-Aguilar y col., (2007) en mango mínimamente procesado. Este comportamiento se atribuye a la oxidación del ácido ascórbico por efecto de la luz UV-C con el incremento de la dosis de radiación.

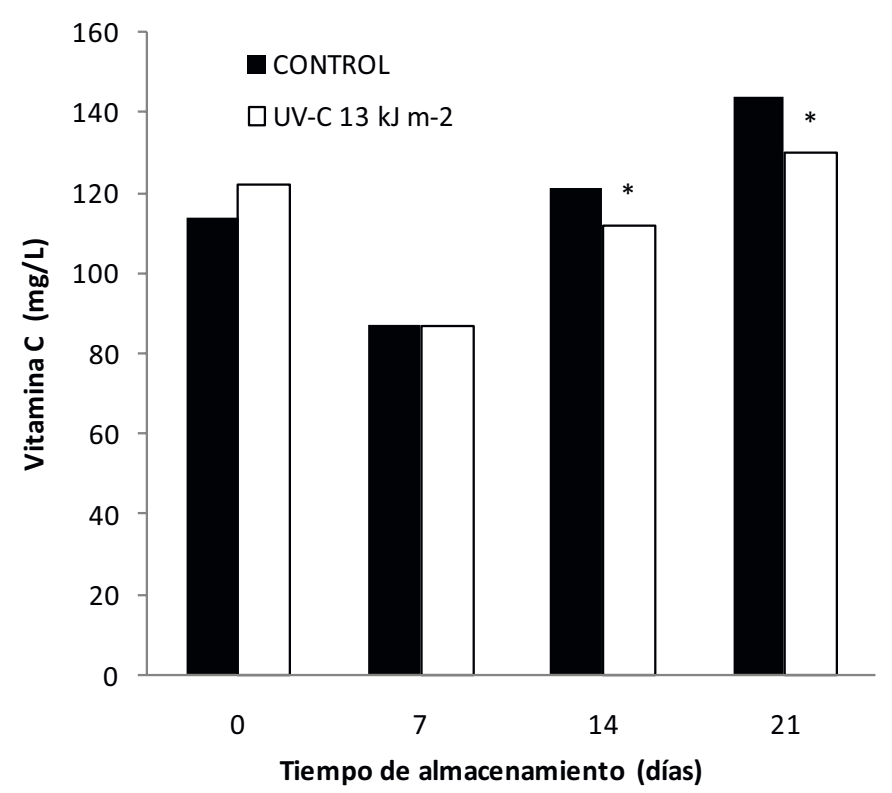

Figura 9. Contenido de Vitamina C en carambola mínimamente procesada control y tratada $\left(13 \mathrm{~kJ} / \mathrm{m}^{2}\right)$ durante 21 días de almacenamiento a $5^{\circ} \mathrm{C}$. El asterisco indica que el valor es significativamente diferente del correspondiente control con una $\mathrm{p}$ $<0.05$.

Tanto frutos tratados como controles presentaron disminución en el contenido de vitamina C en el día 7 a partir del cual se produjo un incremento hasta el día 21 siendo éste mayor en los frutos controles que en los tratados (figura 9). El incremento en la concentración de vitamina $\mathrm{C}$ tanto en frutos tratados como en controles observada a partir del día 7, puede ser consecuencia del balance entre los procesos de biosíntesis, regeneración y degradación que ocurren en la célula. En este sentido, la elucidación de esto requeriría la realización de estudios más detallados, y el significado biológico de estos resultados, deben considerarse con precaución ya que resulta de interés conocer por un lado la contribución de la vitamina C a la capacidad antioxidante total en la carambola mínimamente procesada y, por otra parte, el rol de un antioxidante dado ante condiciones de estrés podría ser 
dependiente de su compartimentalización a nivel celular (AndradeCuvi, 2008).

\subsection{Capacidad antioxidante total}

Inmediatamente después del tratamiento (figura 10) se observó mayor capacidad antioxidante total en los frutos tratados que en los controles. Durante el periodo de almacenamiento tanto frutos tratados como controles presentaron un comportamiento similar en cuanto a la disminución de la capacidad antioxidante del tejido, sin embargo los frutos tratados mantuvieron mayor capacidad antioxidante que los controles.

Se puede decir entonces que la radiación UV-C produjo un efecto positivo sobre la capacidad antioxidante de carambola mínimamente procesada.

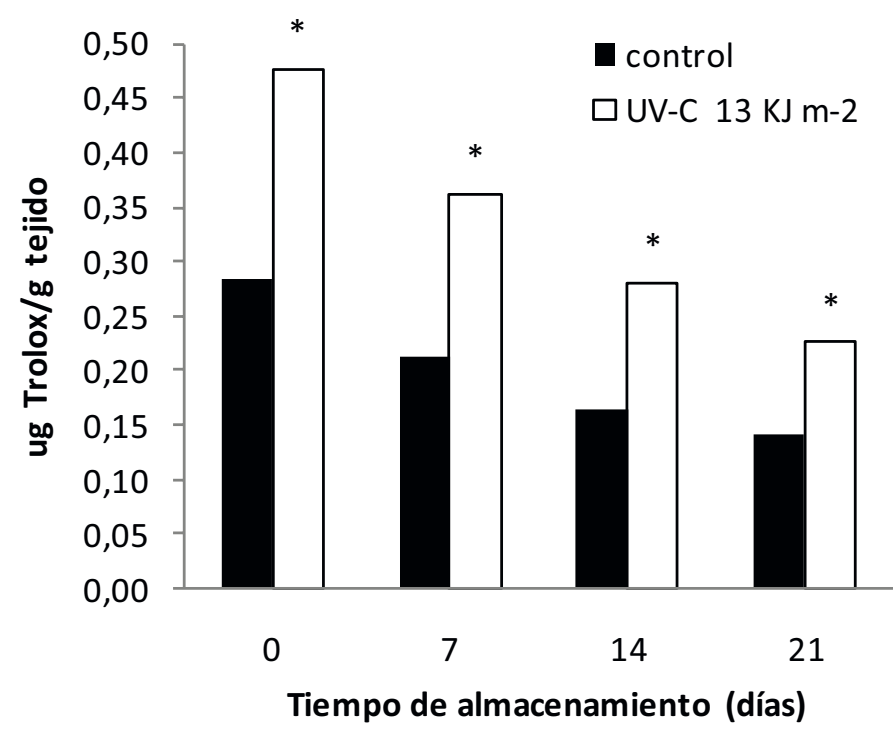

Figura 10. Variación de la capacidad antioxidante total en carambola mínimamente procesada control y tratada $\left(13 \mathrm{~kJ} / \mathrm{m}^{2}\right)$ durante 21 días de almacenamiento a $5^{\circ} \mathrm{C}$. El asterisco indica que el valor es significativamente diferente del correspondiente control con una $\mathrm{p}<0.05$.
La capacidad antioxidante está influenciada principalmente por el contenido de FT, Flav y vitamina C; en el primer caso los frutos tratados presentaron acumulación de estos compuestos. Un parámetro interesante desde el punto de vista nutricional es la capacidad antioxidante de un fruto, los resultados obtenidos en el presente estudio indican que la aplicación de la radiación UV-C como tratamiento poscosecha mantiene la capacidad antioxidante total de la carambola mínimamente procesada durante 21 días, no obstante se produjo una reducción de vitamina $\mathrm{C}$ con respecto a los frutos no tratados.

\section{Medida de la actividad enzimática.}

4.1 Actividad de SOD. La aplicación de algún tipo de estrés (por ejemplo: altas o bajas temperaturas, radiación UV, entre otros.) pueden generar radicales libres que atacan libremente a los lípidos y proteínas de las membranas celulares. Las superóxido dismutasas (SOD) son un grupo de metaloenzimas que catalizan la dismutación de $\mathrm{H}_{2} \mathrm{O}_{2}$ y oxígeno. La inducción de SOD es un importante mecanismo de protección celular bajo una condición de estrés. La actividad de SOD en el día 0 fue mayor en los frutos tratados que en los controles. La mayor actividad de SOD en los frutos control se observó en el día 7 (figura 11), mientras que los frutos tratados presentaron este comportamiento el día 14. Este resultado sugiere que la radiación UV-C retrasó la actividad SOD por 7 días de forma que se aumentaría la eficiencia en la dismutación de $\mathrm{H}_{2} \mathrm{O}_{2}$. 


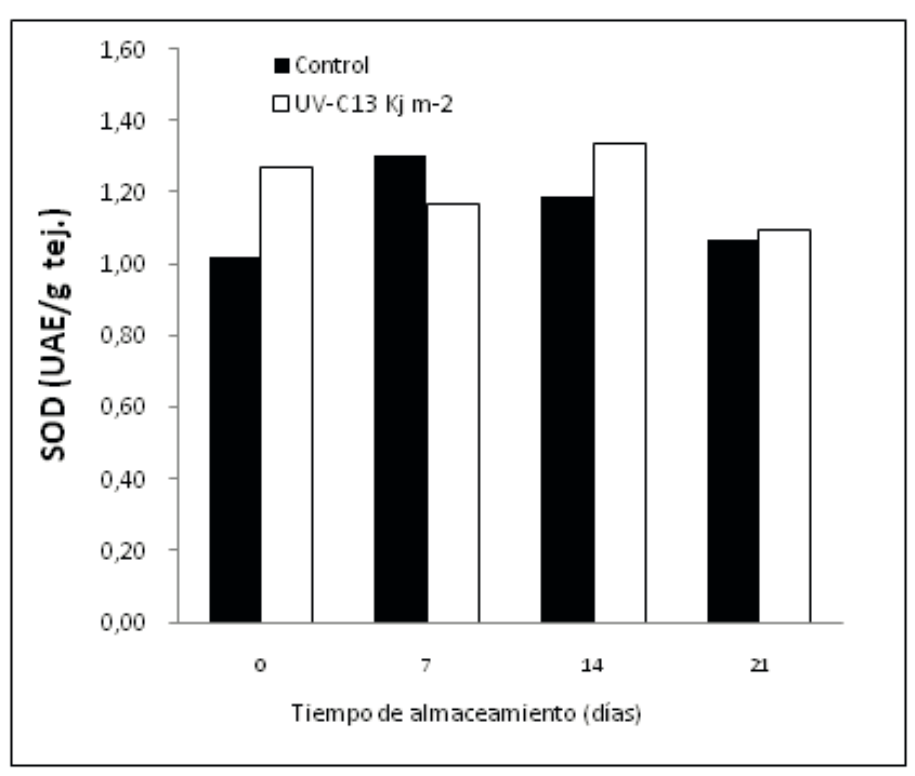

Figura 11. Evolución de la actividad específica de SOD de frutos control y tratados con UV-C (dosis $13 \mathrm{KJ} / \mathrm{m}^{2}$ ) en función del tiempo de almacenamiento a $5^{\circ} \mathrm{C}$. $\mathrm{UAE}_{\mathrm{SOD}}=50 \%$ de inhibición de la reacción.

4.2. Actividad de CAT. La CAT elimina el exceso de $\mathrm{H}_{2} \mathrm{O}_{2}$ producido durante el metabolismo celular evitando su acumulación y consiguiente daño celular. A lo largo del almacenamiento la muestra control presentó perdida de la actividad de CAT, en tanto que en las muestras tratadas este valor se mantuvo constante (figura 12), esto indicaría que la radiación UV-C mantendría activo el sistema antioxidante durante el período de almacenamiento.

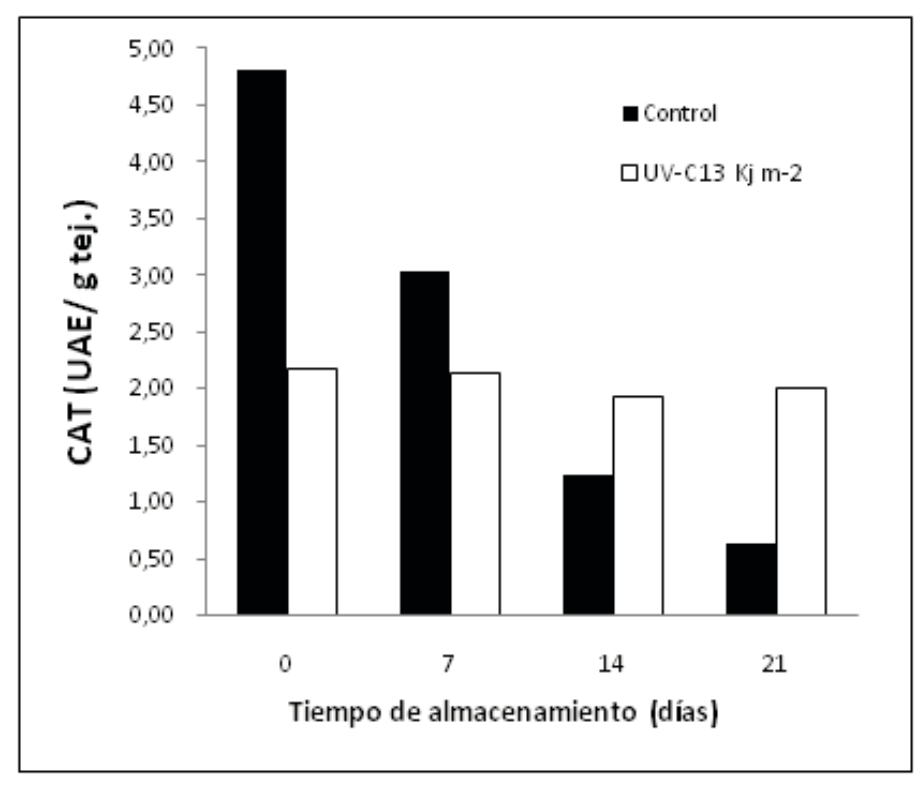

Figura 12. Evolución de la actividad específica de CAT de frutos control y tratados con UV-C (dosis $13 \mathrm{KJ} / \mathrm{m}^{2}$ ) en función del tiempo de almacenamiento a $5^{\circ} \mathrm{C}$. UAE $E_{C A T}=$ mmoles de $\mathrm{H}_{2} \mathrm{O}_{2}$ consumidos/(min. g tejido fresco).

4.3. Actividad de POX. POX es una enzima que tiene diferentes funciones en las plantas superiores incrementando su actividad en respuesta al estrés. Durante el período de almacenamiento la actividad de POX disminuyó tanto en los frutos tratados como en los frutos control, se observó que existe diferencia entre la muestras en el día 21 (figura 13). 


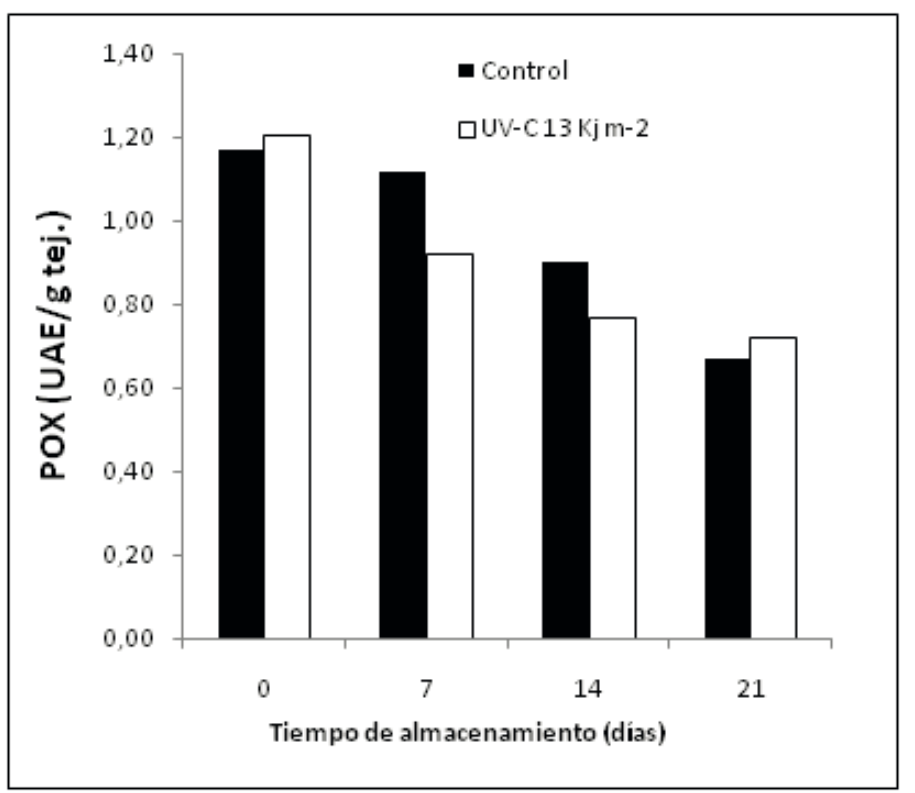

Figura 13. Evolución de la actividad específica de POX de frutos control y tratados con UV-C (dosis $13 \mathrm{KJ} / \mathrm{m}^{2}$ ) en función del tiempo de almacenamiento a $5^{\circ} \mathrm{C}$. $U A E_{\mathrm{POX}}=\Delta \mathrm{OD} /(\mathrm{min}$. g tejido fresco $)$.

Este comportamiento indicaría que la acumulación de $\mathrm{H}_{2} \mathrm{O}_{2}$, generados por SOD que según Alscher y col. (2002) es la primera línea de defensa frente al estrés, estaría siendo eliminada por CAT y otra enzimas como ascorbato peroxidasa y glutatión reductasa en diferentes compartimentos celulares, lo que provocaría un incremento de POX solamente en tiempos largos de almacenamiento indicando una complementariedad en la actividad enzimática antioxidante.

\section{Conclusiones}

La radiación UV-C $\left(13 \mathrm{~kJ} / \mathrm{m}^{2}\right)$ prolongó en 7 días la vida útil de la carambola mínimamente procesada almacenada en refrigeración; produjo menor pérdida de electrolitos y retraso en la aparición de síntomas de daño. Existieron cambios en los parámetros de color $\mathrm{H}$, S y L. Los resultados encontrados en la reducción del crecimiento de mohos proponen a la radiación UV-C como una alternativa tecnológica que retarda el crecimiento de microorganismos. El tratamiento UV-C no influyó sobre los parámetros físico químicos del producto y contribuyó a la acumulación de FT; mantuvo mayores valores de la capacidad antioxidante durante el tiempo de almacenamiento, a pesar de que se produjo disminución en el contenido de vitamina $C$. Al final del almacenamiento, la actividad enzimática de las enzimas analizadas se redujo respecto al día inicial, existió diferencia entre la muestra control y tratadas para SOD (4\%) CAT (31\%) y POX (7\%) indicando que la luz UV-C habría activado el sistema enzimático antioxidante. Sin embargo, resulta de interés realizar más estudios para poder avanzar en la comprensión de las respuestas fisiológicas asociadas con la exposición de tejidos vegetales a la radiación UV-C.

\section{Agradecimientos}

Dirección de Investigación y Transferencia Tecnológica ITT. Proyecto de investigación: Influencia del tratamiento UV-C sobre el tiempo de vida útil y propiedades antioxidantes de productos de IV Gama (mínimamente procesados) de carambola (Averrhoa carambola L.). Facultad de Ciencias de la Ingeniería. Universidad Tecnológica Equinoccial. Quito-Ecuador 


\section{Referencias}

Alscher, R. G.; Erturk, N. y Heath, L. S., (2002). Role of superoxide dismutase (SODs) in controlling oxidative stress in plants, J. Exp. Bot. 53, 1331-1341.

Andrade-Cuvi, M.J. (2008) Relación entre la capacidad antioxidante y el desarrollo del daño por frío en pimientos. Efecto de la radiación UV-C. Tesis. Facultad de Ciencias Exactas. Universidad Nacional de La Plata

Barka, E. A. (2001) Protective enzymes against reactive oxygen species during ripening of tomato (Lycopersicon esculentum L.) fruits in response to low amounts of UV-C. Aust. J. Plant Physiol. No. 28; 785-791

Barka, E. A., Kalantari, S., Makhlouf, J. y Arul, J. (2000) Effects of UV-C irradiation on lipid peroxidation markers during ripening of tomato (Lycopersicon esculentum L) fruits. Aust. J. Plant Physiol. No. 27; 147-152.

Cisneros-Zevallos, L. (2003) The use of controlled postharvest abiotc stresses as a tool for enhancing the nutraceutical content and adding-value of fresh fruits and vegetables. J. of Food Sc.. No. $68 ; 1560-1564$

Concellón, A.; Añón, M.C. y Chaves, A.R. (2005) Effect of chilling on ethylen production in eggplant fruit. Food Chemistry 92: 6369

Costa, L., Vicente, A. R., Civello, P. M., Chaves, A. R. y Martínez, G. A. (2006) UV-C treatment delays postharvest sescence in brócoli florets. Posth. Biol. and Techn. No. 39; 204-210

D’hallewing G., Schirra, M., Manueddu, M., Piga, S. y Ben-Yehoshua, S. (1999) Scoparone and scopoletin accumulation and ultraviolet- $C$ induced resistance to postharvest decay in oranges as influenced by harvest date. J. Am. Soc. Hort. Sci. No. $124 ; 702-707$.
Erkan, M.; Yi Wang, C. y Krizek, D.T. (2001) UV-C irradiation reduces microbial populations and deterioration in Cucurbita pepo fruir tissue. Enviromental and Experimental Botany. 45:1-9

Flurkey, W.H. y Jen, J.J. (1978). Peroxidase and polyphenoloxidase activities in developing peaches. J. Food Sci. 43, 1826-1828.

Giannopolitis, C.N. y Ries, S.K. (1977). Superoxide dismutase I. Occurrence in higher plants. Plant Physiol. 59, 309-314.

González-Aguilar, G. A., Wang, C. y Buta, G. (2004) UV-C irradiation reduces breakdown and chilling injury of peaches during cold storage. Jour. of fhe Sc. Of Food and Agric. 84 ·5 · 415-442

González-Aguilar, G., Zavaleta-Gatica, R. y Tiznado-Hernández, M.E. (2007) Improving postharvest quality of mango 'Haden' by UV-C treatment. Posth. Biol. And Techn. 45 .1. 108-116

González-Aguilar, G.A., Wang, C.Y., Buta, J.G. y Krizek, D.T. (2001) Use of UV-C irradiation to prevent decay and maintain postharvest quality of ripe "Tommy Atkins" mangoes. Int. J. Food Sci. Tech. $36,767-773$

Guerrero-Beltrán J.A. y Barbosa-Cánova, G.V. (2004) Review: Advantages and Limitations on Processing Foods by UV Light. Food Sci Tech Int; 10(3):0137-11

Lamikanra, O., Kueneman, D., Ukuku, D. y Bett-Garber, K. (2005) Effect of Processing Under Ultraviolet Light on the Shelf Life of Fresh-Cut Cantaloupe Melon. Journal of Food Science. No. 70; 534-539

Lemoine, L.M., Civello, P.M., Martínez, G. y Chaves, A.R. (2007) Influence of postharvest UV-C treatment processed broccoli (Brassica oleracea var. J Sci Food Agric 87:1132-1139

Liu, J.; Stevens, C.; Khan, V.A. y Kabwe, M. (1991). The effect of ultraviolet irradiation on shelf-life and ripening of peaches and apples. J. Food Qual. 14, 299-305 
Lobo, M., y González, M. (Sin fecha). Estado actual de los productos mínimamente procesados en España. Laboratorio de Fisiología Vegetal .Dpto. Fruticultura Tropical., Instituto Canario de Investigaciones Agrarias, La Laguna, Tenerife, Islas Canarias, España.

Luckey, T.D. (1980). Hormesis with ionizing radiation, CRC press, Boca Raton.

Marquenie, D., Michiels, C. W., Geeraerd, A. H., Schenk, A., Soontjens, C., Van Impe, J. F., Nikolai, B. M. (2002). Using survival analysis to investigate the effect of UV-C and heat treatment on storage rot of strawberry and sweet cherry. Internatl. J. Food Microbiol. 73: 187-196.

Pan, J., Vicente, A.R.; Martínez, G.A., Chaves, A.R. y Civello, P.M. (2004) Combined used of UV-C irradiation and heat treatment to improve postharvest life of strawberry fruit. Journal of the Science of Food and Agriculture 84:1-9

Piga, A., D’hallewin, G., D'aquino, S. y Agabbio, M. Influence of film wrapping and UV irradiation on cactus pear quality after storage. Packaging Technology and Science. No. 10; 1998: 59-68

Shin, Y.; Hai Liu, R.; Nock, J.F.; Holliday, D. y Watkins, C. (2007). Temperature and relative humidity effects on quality, total ascorbic acid, phenolics and flavonoid concentrations, and antioxidant activity of strawberry. Postharvest Biol. Technol 45, 349-357.

SIGAGRO. (2002). Ecuador Estimación de la producción. Frutas, oleaginosas, fibras, cabuya, bebidas, té y otros cultivos. http:// www.sica.gov.ec/agro/docs/CUADRO.htm

Singleton, V.L. y Rossi, Jr. J.A. (1965). Colorimetry of total phenolics with phosphomolybidic-phosphotungstic acid reagents. Am. J.Enol. Vitic.16, 144-158.

Sinha, A.K. (1972) Colorimetric Assay of Catalase. Analytical Biochemistry 47, 389-394
Vicente, A., Pineda, C., Lemoine, L., Civello, P., Martínez, G., Chaves, A. (2005) UV-C treatments reduce decay, retain quality and alleviate chilling injury in pepper. Postharvest Biol. Technol. No. $35 ; 69-79$.

Wang, C.Y. (1995) Effect of temperature preconditioning on catalase, on refrigerated storage of minimally peroxidase, and superoxide dismutase in chilled zucchini squash. Postharvest Biology and Technology 5 67-76 
